\title{
SINGLE-USER PERFORMANCE OF UPLINK DS-CDMA USING RELAY-ASSISTED DIVERSITY
}

\author{
Wei Fang, Lie-Liang Yang and Lajos Hanzo \\ School of ECS, University of Southampton, SO17 1BJ, UK
}

\section{ABSTRACT}

In a wireless system with distributed mobile users, several mobile users may cooperate with each other in order to achieve the transmit diversity. In this contribution we investigate the single-user performance of an uplink direct-sequence codedivision multiple-access (DS-CDMA) scheme, where one user is assisted by several other users (relays) so as to achieve the relay-assisted diversity. Specifically, the performance of the relay-assisted DS-CDMA is investigated, when communicating over generalized Nakagami- $m$ fading channels, where signals from the transmitter to the relays and that from the relays to the base-station (BS) receiver may experience different fading. In our study two types of detection schemes are invoked. The first detection scheme is a maximal ratio combining (MRC) assisted single-user detection (SUD) scheme, which maximizes the output signal-to-noise ratio (SNR) without taking the interference among the relays into account. By contrast, the second detection scheme maximizes the output signalto-interference-plus-noise ratio (MSINR), which is a multiuser detection (MUD) scheme that is capable of suppressing the interference among the relays. Our study and results show that, in a relay-assisted DS-CDMA without using orthogonal spreading codes, the relay-assisted diversity may only be achievable after suppressing the inter-relay interference.

\section{INTRODUCTION}

It is well-known that transmission over wireless channels suffers from fading, which may be mitigated by exploiting various types of diversity in time-, frequency- and/or space-domain. Specifically, in space-domain the spatial diversity can be obtained by transmitting the same signal from geographically separated transmitters, thus generating independently faded replicas of the signal at the receiver. In wireless communications spatial diversity is particularly attractive, since it offers spectral efficiency without incurring an expenditure of transmission time or bandwidth [1]. In practice multiple-antennas are desirable for deployment at cellular base stations, in order to achieve the downlink transmit diversity. However, the transmit diversity depending on multiple transmit antennas is not directly applicable to the uplink transmission, due to the mobile unit's size. Recently, cooperative diversity in wireless systems with distributed nodes or users has attracted wide attention $[2,3]$. This is because the capacity of a wireless system may be significantly improved through cooperation among the distributed nodes or users. Specifically, in a wireless system with distributed mobile users, a set of mobile users may share their transmit antennas in order to create a virtual antenna array for achieving the transmit diversity [2-5]. This type of transmit diversity is usually referred to as relay-assisted diversity.
The performance of the relay-assisted diversity scheme has been investigated in $[4,5]$, when assuming that there exists no interference amongst the relays. However, in practice, for example in cellular DS-CDMA sy stems, there usually exists multiuser interference among the mobile users. Hence, in this contribution we investigate the bit-error-rate (BER) performance of an uplink DS-CDMA scheme, where one user is assisted by several other users acting as relays, so as to achieve the relay-assisted diversity. In our study, we assume a generalized Nakagami channel mode1 [8], where signals from the transmit"ter to the relays and that from the relays to the BS receiver may experience different fading. By contrast, [4] assumed only Rayleigh fading, while [5] considered a common Nakagami- $m$ fading for both the transmitter-relay and relay-receiver channels. Furthermore, in this contribution two types of detection schemes are investigated. The first detection scheme is a SUD, which maximizes the output SNR without taking the interference among the relays into account. By contrast, the second detection scheme is a MUD, which maximizes the output SINR by taking both noise and interference among the relays into account.

Note that, the reason for us focusing our attention only on single-user (multiple relays) case is that from the single-user performance results we can gain insight into the achievable performance, when the DS-CDMA system supports multiple users associated with employing advanced MUD. Furthermore, it can be shown that our approaches in this paper can be readily extended to the multi-user cases.

\section{System Description}

\section{A. Transmitted Signal}

In our considered DS-CDMA there is one mobile user, say user $k$, communicating with the BS with the assistance of $L$ relays, which are also mobile users. The transmitter schematic of user $k$ is shown in Fig. 1. It can be shown that the signal transmitted. by the $k$ th user can be expressed as

$$
s_{k}(t)=\sqrt{2 P_{k}} b_{k}(t) c_{k}(t) \cos \left(2 \pi f_{c} t+\phi_{k}\right)
$$

where $P_{k}$ represents the transmitted power of user $k, f_{c}$ is the carrier frequency, while $\phi_{k}$ denotes the initial phase angle associated with the carrier modulation. In (1) $b_{k}(t)$ represents the transmitted data waveform, which can be expressed as

$$
b_{k}(t)=\sum_{n=0}^{\infty} b_{k}[n] P_{T_{b}}\left(t-n T_{b}\right)
$$

where $b_{k}[n] \in\{-1,+1\}, T_{b}$ represents the bit duration, $P_{T_{b}}(t)$ represents the rectangular waveform, which is defined 


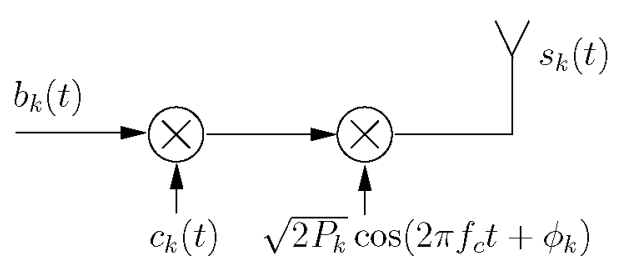

Figure 1: Transmitter schematic block diagram of the $k$ th user.

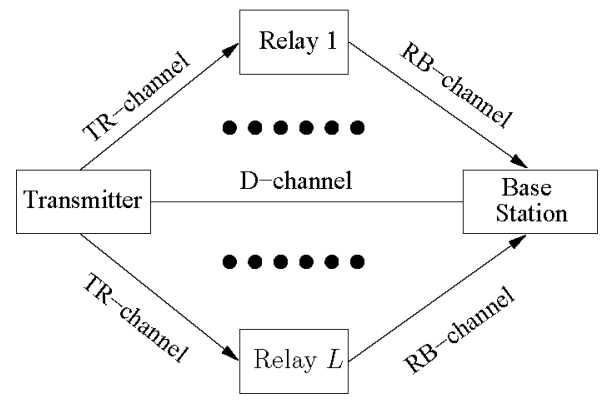

Figure 2: Channels in a relay-assisted DS-CDMA, where one transmitter is assisted by $L$ relays.

as $P_{T_{b}}(t)=1$ if $0 \leq t<T_{b}$, and $P_{T_{b}}(t)=0$ otherwise. Furthermore, in (1), $c_{k}(t)$ represents the DS spreading waveform, which can be expressed as

$$
c_{k}(t)=\sum_{n=0}^{\infty} c_{k n} \psi_{T_{c}}\left(t-n T_{c}\right)
$$

where $T_{c}$ represents the chip-duration, $N=T_{b} / T_{c}$ represents the spread factor, $c_{k n} \in\{-1,+1\}, \psi_{T_{c}}(t)$ is the chipwaveform, which is defined within $\left[0, T_{c}\right)$ and normalized to satisfy $\int_{0}^{T_{c}} \psi_{T_{c}}^{2}(t) d t=1$. Let us now describe the cooperation scheme.

\section{B. Cooperation Operation}

We assume that there are $L$ relays constituted by the mobile users, which assist the transmission from user $k$ to the BS, as shown in Fig. 2. For the sake of comenience, in Fig. 2 we define the direct (D) channel as the D-channel, which directly connects user $k$ with the BS. The relay chamel is defined as the $\mathrm{R}$-channel, which represents the channel from user $k$ through a relay to the BS. Furthermore, the R-channel includes the channel connecting the $k$ th user to the relay and that connecting the relay to the BS. Hence, for convenience, the former is referred to as the TR-channel, while the latter as the RB-channel.

Throughout this paper we assume that a mobile user cannot transmit and receive signal simultaneously, and the cooperation scheme is based on time-division (TD). Specifically, in the considered cooperation scheme, user $k$ communicates with the $L$ relays and the $B S$ receiver over the first time-slot, while within the second time-slot, the $L$ relays transmit the signal received from user $k$ in the first time-slot to the BS. Furthermore, in this paper we assume frequency non-selective fading channels so as to focus on relay-assisted diversity alone. Additionally, we assume that transmitted signals can be perfectly synchronized whenever necessary.

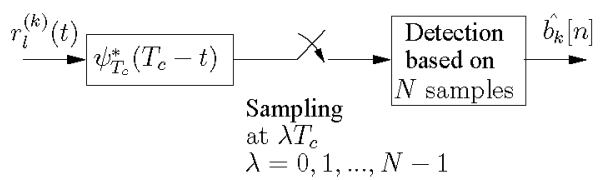

Figure 3: Receiver schematic diagram of the relays.

Let the complex baseband equivalent signal received by the th relay within the first time-slot of the $n$th bit duration be expressed as

$$
r_{i}^{(k)}(t)=\sqrt{2 P_{k}} h_{l}^{(k)} b_{k}[n] c_{k}(t)+n_{l}^{(k)}(t), l=1,2, \ldots, L
$$

where $h_{l}^{(k)}$ represents the fading gain of the lth TR channel, while $n_{l}^{(k)}(t)$ represents the complex baseband equivalent Gaussian noise, which has mean zero and a variance of $N_{0}$ per dimension. The received signal of (4) is first detected by the th relay on a symbol-by-symbol basis according to Fig. 3 , in order to generate a soft estimate, $\hat{b}_{k}[n]$ for the transmitted bit $b_{k}[n]$. Specifically, as shown in Fig. $3, r_{l}^{(k)}(t)$ is first input to a filter matched to the transmitted chip-waveform $\psi_{T_{c}}(t)$. Then, the matched-filter's output is sampled at the chip-rate, which provides $N$ samples per symbol to the $l$ th detector. According to Fig. 3, given the knowledge of $h_{l}^{\left({ }^{k}\right)}$, the $\lambda$ th sample can be expressed as

$$
\begin{array}{r}
y_{i \lambda}=\frac{1}{\sqrt{2 P_{k} N T_{c}}} \int_{\lambda T_{c}}^{(\lambda+1) T_{c}} r_{l}^{(k)}(t) \psi_{T_{c}}^{*}(t) d t \\
\lambda=0,1, \cdots, N-1
\end{array}
$$

Upon substimting (4) into (5), we obtain

$$
y_{l \lambda}=\frac{1}{\sqrt{N}} h_{l}^{(k)} b_{k}[n] c_{k \lambda}+N_{l \lambda}, \lambda=0,1, \cdots, N-1
$$

where $N_{l \lambda}$ is the Gaussian noise component, which is given by

$$
N_{l \lambda}=\frac{1}{\sqrt{2 P_{k} N T_{c}}} \int_{\lambda T_{c}}^{(\lambda+1) T_{c}} n_{l}^{(k)}(t) \psi_{T_{c}}^{*}(t) d t
$$

which has mean zero and a variance of $N_{0} / 2 E_{k}$ per dimension, where $E_{k}=P_{k} T_{b}$ represents the energy per bit received by the th relay from the $k$ th transmitter.

Let us define

$$
\begin{aligned}
y_{l} & =\left[y_{l 0}, y_{l 1}, \cdots, y_{l(N-1)}\right]^{T} \\
N_{l} & =\left|N_{l 0}, N_{l 1}, \cdots, N_{l(N-1)}\right|^{T} \\
c_{k} & =\frac{1}{\sqrt{N}}\left[c_{k 0}, c_{k 1}, \ldots, c_{k(N-1)}\right]^{T}
\end{aligned}
$$

Then, it can be shown that we have

$$
y_{l}=c_{k} h_{l}^{(k)} b_{k}[n]+N_{l}
$$

From (9), we can readily obtain the estimate $\hat{b}_{k}[n]$, which can be expressed as

$$
\hat{b}_{k}[n]=\frac{1}{h_{l}^{(k)}} c_{k}^{T} \boldsymbol{y}_{l}=b_{k}[n]+\frac{1}{h_{l}^{(k)}} c_{k}^{T} \boldsymbol{N}_{l}
$$


Explicitly, $\hat{b}_{k}[n]$ contains noise in addition to the bit $b_{k}[n]$ to be relayed.

After the detection, $\hat{b}_{k}[n]$ is then spread and relayed by the th relay to the BS within the second time-slot of the nth bit duration by using the similar scheme shown in Fig.1. Correspondingly, the transmitted signal of the th relay can be expressed as

$$
\begin{array}{r}
s_{l}^{(k)}(t)=\sqrt{2 P_{k l}} \hat{b}_{k}[n] c_{l}^{(k)}(t) \cos \left(2 \pi f_{c} t+\phi_{l}^{(k)}\right), \\
l=1,2, \cdots, L
\end{array}
$$

where $P_{k l,} c_{l}^{(k)}(t)$ and $\phi_{l}^{(k)}$ represent, respectively, the transmitted power, signature waveform and initial phase associated with the the $l$ th relay signal.

Consequently, at the BS, the received complex baseband equivalent signal within the first time-slot of the $n$th bit duration can be expressed as

$$
r_{0}(t)=\sqrt{2 P_{k}} h_{0}^{(k)} b_{k}[n] c_{k}(t)+n(t)
$$

where $h_{0}^{(k)}$ represents the channel gain of the D-channel, while $n(t)$ the Gaussian noise received at the BS, which, again, has zero mean and a variance of $N_{0}$ per dimension. The received complex baseband equivalent signal by the BS during the second time-slot of the $n$th bit duration can be expressed as

$$
\begin{aligned}
r_{1}(t)= & n(t)+\sum_{l=1}^{L} \sqrt{2 P_{k l}} h_{r l}^{(k)} b_{k}[n] c_{l}^{(k)}(t) \\
& +\sum_{l=1}^{L} \sqrt{2 P_{k l}} h_{r l}^{(k)}\left[\frac{c_{k}^{T} N_{l}}{h_{l}^{(k)}}\right] c_{l}^{(k)}(t)
\end{aligned}
$$

\section{Channel Modeling}

We assume that the TR-channels and RB-channels may experience different fading. Specifically, let in (13) $h_{l}^{(k)}=\alpha_{l 1} e^{j \theta_{l 1}}$ and $h_{r l}^{(k)}=\alpha_{l 2} e^{j \theta_{l 2}}$ for $l=1,2, \ldots, L$, where $\alpha_{l 1}, \alpha_{l 2}$ and $\theta_{l 1}, \theta_{l 2}$ signify the amplitudes and phases of the $l$ th TR-channel and RB-channel, respectively. We assume that the fading amplitude $\alpha_{i i}, i=1,2$, obeys the Nakagami distribution with the probability density functions (pdI) of

$$
f\left(\alpha_{l i}\right)=\frac{2 m_{l i}^{m_{i i}} \alpha_{l i}^{2 m_{l i}-1}}{\Gamma\left(m_{l i}\right) \Omega_{l i}} e^{-\left(m_{l i} / \Omega_{l i}\right) \alpha_{l i}^{2}}, i=1,2
$$

where $m_{l,}$ represents the fading parameter of the $l$ th TRchannel when $i=1$ or the $l$ th $\mathrm{RB}$-channel corresponding to $i=2$. According to [8], $m_{l i}=E^{2}\left[\alpha_{l i}^{2}\right] / \operatorname{Var}\left[\alpha_{l i}^{2}\right]$. In (14) $\Omega_{l i}$ is a scaling parameter that signifies the average power, which was assumed to be unity in our simulations. Note that $h_{0}=\alpha_{0} e^{j \theta_{0}}$, where $\alpha_{0}$ and $\theta_{0}$ signify the amplitude and phase of the Dchannel. The pdf of $\alpha_{0}$ can be readily obtained from (14) with $m_{i,}$ replaced by $m_{0}$ and $\Omega_{i,}$ by $\Omega_{0}$. Furthermore, the phases $\theta_{0}$ and $\theta_{l i}$ for $l=1,2, \ldots, L$ are assumed to be the independent identically distributed (iid) random variables uniformly distributed within $[0,2 \pi)$. Let us now considering the deteclion algorithms.

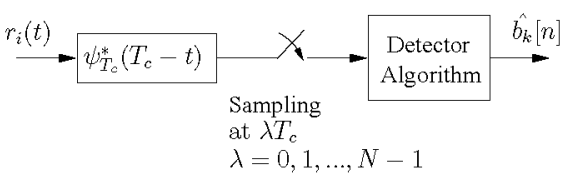

Figure 4: Receiver schematic block diagram at the base station (BS)

\section{Detection AlgorithMS}

In this section we investigate the detection of the relay-assisted DS-CDMA signal, when assuming that the spreading codes of the transmitter and relays constitute the set of sequences existing cross-correlations. Hence, there exists interference among the relayed signals, since these relayed signals are received by the BS at the same time. Specifically, two types of detection schemes are derived, which are detailed in our forthcoming discourse. Let us first derive the representation of the received signal by the BS.

\section{A. Representation of the BS Received Signal}

As at the relays, the received signal at the $B S$ is first filtered by a chip-waveform matched-filter and is then sampled at chip-rate in order to provide the detector observation samples, as shown in Fig. 4 . Since $b_{k}[n]$ is transmitted by two time-slots, each of which is associated with $N$-length spreading sequences, hence the BS receiver can obtain a total of $2 N$ samples for detecting $b_{k}[n]$. Let $\mathbf{y}=\left[\mathbf{y}_{0}^{T}, \mathbf{y}_{1}^{T}\right]^{T}$ contain the $2 N$ observation samples, where $y_{i}=\left[y_{i 0}, y_{i 1}, \cdots, y_{i(N-1)}\right]^{T}, i=0,1$. Assuming $P_{k}=P_{k l}=P_{0}$, then it can be shown that $y_{i \lambda}$ can be expressed as

$$
\begin{array}{r}
y_{i \lambda}=\frac{1}{\sqrt{2 P_{0} N T_{c}}} \int_{\lambda T_{0}}^{(\lambda+1) T_{c}} r_{i}(t) \psi_{T_{c}}^{*}(t) d t, i=0,1 \\
\lambda=0,1, \cdots, N-1
\end{array}
$$

Upon substituting (12) or (13) into the above equation, we obtain

$$
\begin{gathered}
y_{0 \lambda}=h_{0}^{(k)} c_{k \lambda} b_{l}[n]+n_{0 \lambda} \\
y_{1 \lambda}=\sum_{l=1}^{L} h_{r l}^{(k)} c_{l \lambda}^{(k)} b_{k}[n]+\sum_{l=1}^{L} h_{r l}^{(k)} c_{l \lambda}^{(k)}\left[\frac{c_{k}^{T} N_{l}}{h_{l}^{(k)}}\right]+n_{1 \lambda} \\
\lambda=0,1, \cdots, N-1
\end{gathered}
$$

where $n_{i \lambda}, i=0,1$ is an independent Gaussian random variable with zero mean and a variance of $N_{0} / 2 E_{0}$ per dimension, where $E_{0}=P T_{b}$ represents the average energy per bit received from the D-channel or one of the TB-channel.

Let

$$
\begin{aligned}
c_{k i} & =\frac{1}{\sqrt{N}}\left[c_{l 0}^{(k)}, c_{l 1}^{(k)}, \ldots, c_{l(N-1)}^{(k)}\right]^{T} \\
n_{i} & =\left[n_{i 0}, n_{i 1}, \cdots, n_{i(N-1)}\right]^{T}, i=0,1
\end{aligned}
$$

Then, it can be shown that, after ignoring the superscript $k$ for convenience, $y$ can be expressed as

$y=\left[\sum_{l=1}^{c_{k} h_{0}} c_{k l} h_{r l}\right] b_{k}[n]+\left[\sum_{l=1}^{L} c_{k l} h_{r l}\left[\frac{c_{l}^{T} N_{l}}{h_{l}}\right]\right]+\left[\begin{array}{l}n_{0} \\ n_{1}\end{array}\right]$ 
Furthermore, the above equation can be rewritten as

$$
\boldsymbol{y}=\boldsymbol{C} h_{0 L} b_{k}[n]+\underbrace{C_{L} \bar{H}_{r L}\left(\overline{\boldsymbol{I}}_{L} \otimes c_{k}^{T}\right) \boldsymbol{n}_{t r}+\boldsymbol{n}_{r}}_{\boldsymbol{n}_{I}}
$$

where $\otimes$ represents the Kronecker product [7] operation and the other arguments in (19) are given as follows:

$$
\begin{aligned}
\boldsymbol{C} & =\left[\begin{array}{ccccc}
c_{k} & 0 & 0 & \ldots & 0 \\
0 & c_{k 1} & c_{k 2} & \ldots & c_{k L}
\end{array}\right] \\
h_{O L} & =\left[h_{0}, h_{r 1}, h_{r 2}, \cdots, h_{r L}\right]^{T} \\
\boldsymbol{C}_{L} & =\left[\begin{array}{cccc}
0 & 0 & \ldots & 0 \\
c_{k 1} & c_{k 2} & \ldots & c_{k L}
\end{array}\right] \\
\boldsymbol{H}_{r L} & =\operatorname{diag}\left\{\frac{h_{r 1}}{h_{1}}, \frac{h_{r 2}}{h_{2}}, \ldots, \frac{h_{r L}}{h_{L}}\right\} \\
n_{t r} & =\left[N_{1}^{T}, N_{2}^{T}, \cdots, N_{L}^{T}\right]^{T} \\
n_{r} & =\left[n_{0}^{T}, n_{1}^{T}\right]^{T}
\end{aligned}
$$

Having obtained the representation of the BS received signal as seen in (19), let us now consider the detection scheme.

\section{B. Maximal Ratio Combining (MRC) Assisted Single-User Detection}

The received signal $y$ of (19) can be despread using $C^{T}$, yielding

$$
\bar{y}=C^{T} y
$$

It can be shown that the th component of $\bar{y}$ can be expressed as

$$
\bar{y}_{l}= \begin{cases}h_{0} b_{k}[n]+\bar{n}_{0}, & \text { if } l=0 \\ h_{r l} b_{k}[n]+\frac{h_{r l}}{h_{l}} c_{k}^{T} N_{l}+c_{k l}^{T} n_{1}+\mathrm{RII}, & \text { if } l>0\end{cases}
$$

where IRI represents the inter-relay interference.

Since the MRC-assisted SUD scheme does not exploit the knowledge of the inter-relay interference, it can be readily shown that the weight for $\bar{y}_{l}$ is given by

$$
w_{l}= \begin{cases}h_{0}^{*}, & \text { for } l=0 \\ \frac{\left|h_{i}\right|^{2} h_{r l}^{*}}{\left|h_{l}\right|^{2}+\left|h_{r l}\right|^{2}}, & \text { for } l>0\end{cases}
$$

Note that the above-derived combining scheme maximizes the SNR, when there exists no inter-relay interference.

\section{Maximum SINR Assisted Multiuser Detection}

Since the BS receiver employs the knowledge about the spreading codes and channels of both the $k$ th user and its relays, the SINR can jointly be maximized as follow.

Let $w$ be the weight vector, which maximizes the SINR of the combiner. Then, it can be shown that the optimum weight vector is given by [6]

$$
w_{o p t}=\mu R_{I}^{-1} \boldsymbol{H}=\mu \boldsymbol{R}_{I}^{-1} C h_{O L}
$$

where $\mu$ is a constant, $R_{I}=E\left[n_{I} n_{I}^{H}\right]$ is the covariance matrix of $n_{I}$. Correspondingly, the decision variable is given by

$$
z_{n}=\Re\left\{w_{o p t}^{H} \boldsymbol{y}\right\}
$$

where $y$ is shown in (19), while $\Re\{x\}$ represents the real-part of $x$.

\section{PERFORMANCE RESULTS}

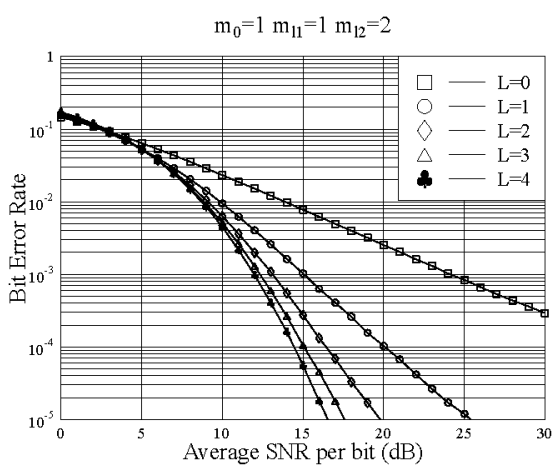

Figure 5: BER performance bound of the relay-assisted DS-CDMA supporting single-user, when the D-channel and the TR-channels experience Rayleigh fading, while the RB-channels experience Nakagami- $m$ fading associated with $m_{l 2}=2.0$ for $l=1,2,3,4$.

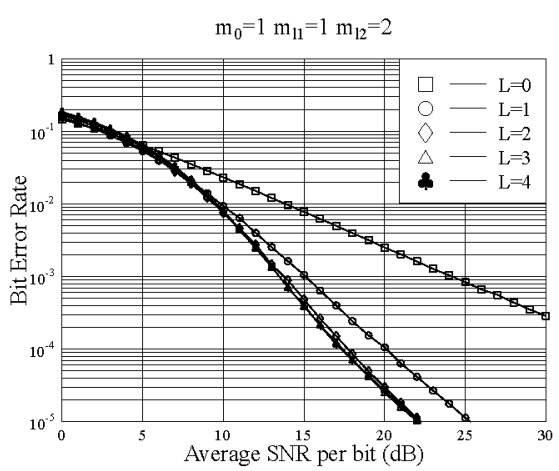

Figure 6: BER versus the SNR per bit performance of the relay-assisted DSCDMA using $m$-sequence and MRC-assisted SUD, when the D-channel and the TR-channels experience Rayleigh fading, while the RB-channels experience Nakagami- $m$ fading associated with $m_{l 2}=2.0$ for $l=1,2,3,4$.

In this section we provide a range of simulation results in order to illustrate the BER versus the SNR per bit performance for the relay-assisted cooperative DS-CDMA systems, when communicating over Nakagami- $m$ fading channels. In our simulations we assumed that power control was employed, resulting in that the received power from the original transmitter and that from any of the relays were the same. Furthermore, in order to carry out a fair comparison, the average SNR associated with one transmitted data bit was maintained to be $E_{b} / N_{0}$ regardless of the value of $L$.

Fig.5 shows the BER versus the average SNR per bit performance bound of the proposed relay-assisted DS-CDMA, when the D-channel and the TR-chamels experience Rayleigh fading, while the RB-channels experience Nakagami- $m$ fading associated with $m_{l 2}=2.0$ for $l=1,2,3,4$. In our simulations orthogonal spreading codes were employed, hence there was no interference among the relays. Consequently, the BER performance shown in Fig. 5 represents the best BER perfor- 


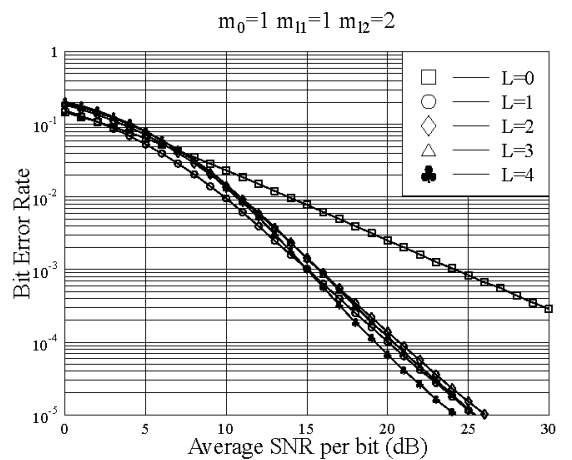

Figure 7: BER versus the SNR per bit performance of the relay-assisted DS-CDMA using random spreading sequences and MRC-assisted SUD, when the $\mathrm{D}$-channel and the TR-channels experience Rayleigh fading, while the RBchannels experience Nakagami-m fading associated with $m_{l 2}=2.0$ for $l=$ $1,2,3,4$.

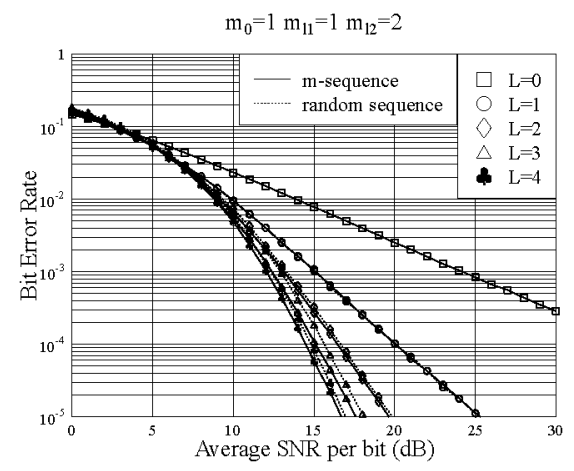

Figure 8: BER versus the SNR per bit performance of the relay-assisted DS-CDMA using both $m$-sequences and random spreading sequences and MSINR-assist ed MUD, when the D-chamel and the TR-channels experience Rayleigh fading, while the RB-channels experience Nakagami- $m$ fading associated with $m_{l 2}=2.0$ for $l=1,2,3,4$

mance that the relay-assisted DS-CDMA is capable of achieving. The results of Fig.5 show that the BER performance improves, when using more relays for providing the diversity, provided that the average SNR per bit is sufficiently high. However, as shown in Fig.5, if the average SNR per bit is too low, diversity gain cannot be guaranteed and the BER performance may even become worse, when using more relays.

In Fig. 6 and Fig. 7 we show the BER versus the average SNR per bit performance of the relay-assisted DS-CDMA associated with using the MRC-assisted SUD derived in Section B.. Specifically, in the context of Fig.6 the spreading codes were constituted by the $m$-sequences, while in Fig. 7 they were constituted by random sequences. From the results of Figs. 6 and 7 we observe that the BER performance in both figures is the same as that shown in Fig. 5 , when $L=1$. The reason for this is that, when $L=1$, there exists no interference between user $k$ and its relay due to using time-division. However, as shown in Figs. 6 and 7 , when $L \geq 2$, we observe that there is either small diversity gain or no diversity gain at all. This is because, when
$L \geq 2$, there exists significant interference among the relays when using the MRC-assisted SUD.

Finally, Fig. 8 shows the BER versus the average SNR per bit performance for the relay-assisted DS-CDMA associated with using the the MSINR-assisted MUD derived in Section C., when communicating over generalized Nakagami- $m$ fading channels. The parameters used in our simulations for this figure were the same as that used in Figs.5, 6 and 7. Both $m$ sequences and radom spreading sequences were considered. From the results of Fig.8, explicitly, the BER performance corresponding to any of the cases is close to the BER performance bound shown in Fig.5. Therefore, when the MSINR-assisted MUD is employed, the interference among the relays can be efficiently mitigated.

In summary, in this contribution we have investigated the performance of a relay-assisted DS-CDMA, when communicating over generalized Nakagami- $m$ fading channels. From our study and simulation results, we conclude that in a relayassisted DS-CDMA without using orthogonal spreading codes, the relay-assisted diversity may only be achievable when the interference among the relays is efficiently suppressed.

\section{ACKNOWLEDGMENT}

The author would like to acknowledge with thanks the financial assistance from EPSRC of UK.

\section{REFERENCES}

[1] R. U. Nabar and H. Bölcskei, "Space-time signal design for fading relay channels," Proc. IEEE Globecom, San Francisco, CA, Vol. 4, pp. 19521956, Dec. 2003.

[2] A. Sendonaris, E. Erkip, and B. Aazhang, "Increasing uplink capacity via user cooperation diversity," in Proc. of IEEE Int. Symp. on Info. Theory, Cambridge, MA, Ang. 1998, p. 156.

[3] A. Sendonaris, E. Erkip, and B. Aazhang, "User cooperation diversity Part I and II," IEEE Transactions on Wireless Communications, Vol. 51, No. 11, Nov. 2003.

[4] P. A. Anghel and M. Kaveh, "Exact symbol error probability of a cooperative network in a Rayleigh-fading environment," IEEE Transactions on Wireless Communications, Vol. 3, No. 5, pp. 1416-1421, Sep. 2004.

[5] M. O. Hasna and M.-S. Alouini, "Harmonic mean and end-to-end performance of transmission systems with relays," IEEE Transactions on Communications, vol. 52, pp. $130-135$, January 2004.

[6] Compton, R. T. (Jr), Adaptive Antemas: Concepts and Performance. Englewood Cliffs, New Jersey, USA: Prentice-Hall, 1996.

[7] H. L. V. Trees, Optimum Array Processing. Wiley Interscience, 2002.

[8] M. Nakagami, "The m-distribution: a general formula of intensity distribution of radid fading," in Statistical Methods in Radio Wave Propagation. W. C. Hoffman, Ed, New York: Pergamon, 1960, pp, 3-36. 